\title{
Choque em crianças
}

\section{Shock in children}

Ana Paula de Carvalho Panzeri Carlotti

\section{RESUMO}

Este texto apresenta uma revisão da definição, classificação, fisiopatologia e tratamento inicial dos diversos tipos de choque na criança. O reconhecimento precoce e o tratamento agressivo do choque em tempo oportuno são essenciais à prevenção da parada cardiorrespiratória e à melhora do desfecho.

Palavras-chave: Choque. Criança. Diagnóstico. Ressuscitação Hídrica. Suporte Hemodinâmico.

\section{Definição}

Choque é a situação clínica resultante do desequilíbrio entre a oferta de oxigênio e nutrientes e a demanda metabólica dos tecidos. ${ }^{1-4}$

\section{Fisiopatologia}

O choque se caracteriza por déficit agudo de oxigênio nas células, que resulta em metabolismo anaeróbico e acidose láctica. ${ }^{1}$ Para compreensão adequada da fisiopatologia do choque e dos fundamentos de seu tratamento é essencial que se conheça o conceito de transporte de oxigênio.

$\mathrm{O}$ transporte de oxigênio $\left(\mathrm{DO}_{2}\right)$, ou seja, a quantidade de oxigênio transportado ao corpo por minuto, é o produto do conteúdo arterial de oxigênio pelo débito cardíaco (Equação 1).

O conteúdo arterial de oxigênio $\left(\mathrm{CaO}_{2}\right)$, em mL de oxigênio por $\mathrm{dL}$ de sangue, é determinado pela concentração de hemoglobina e sua saturação com oxigênio e, em menor proporção, pela quantidade de oxigênio dissolvido no plasma (Equação 2).

O débito cardíaco é o produto da frequência cardíaca pelo volume sistólico (Equação 3).

$$
\mathrm{DO}_{2}=\text { Conteúdo arterial de } \mathrm{O}_{2}\left(\mathrm{CaO}_{2}\right) \text { x Débito cardíaco (Equação 1) }
$$

$\mathrm{CaO}_{2}=$ Concentração de hemoglobina $(\mathrm{g} / \mathrm{dL})$ x 1,34 x Saturação arterial de $\mathrm{O}_{2}\left(\mathrm{SaO}_{2}\right)+\left(\mathrm{PaO}_{2}\right.$ x 0,003) (Equação 2)

Débito cardíaco = Frequência cardíaca x Volume sistólico (Equação 3)

Professora Associada do Departamento de Puericultura e Pediatria da Faculdade de Medicina de Ribeirão Preto da Universidade de São Paulo
Correspondência: Avenida dos Bandeirantes 3900 14049-900 / Ribeirão Preto - SP. apcarlotti@fmrp.usp.br

Artigo recebido em 11/04/2012 Aprovado para publicação em 20/06/2012 
O volume sistólico é a quantidade de sangue ejetado do coração a cada contração. É determinado por três componentes: pré-carga, contratilidade e póscarga. A pré-carga é a quantidade de estiramento da fibra muscular antes do início da contração, e se relaciona, principalmente, ao volume de enchimento das câmaras cardíacas. O volume de enchimento é o volume contido no ventrículo no final da diástole. $\mathrm{O}$ aumento da pré-carga aumenta a contração muscular pelo mecanismo de Frank-Starling, ou seja, quanto maior o estiramento, maior o volume de ejeção. A contratilidade se refere à força e à eficiência da contração muscular. A pós-carga é a soma das forças que se opõem à ejeção ventricular, determinada pela elasticidade do leito vascular e pela resistência ao fluxo nas artérias e arteríolas. Clinicamente, a resistência vascular sistêmica constitui a pós-carga do ventrículo esquerdo e a resistência vascular pulmonar, a pós-carga do ventrículo direito. ${ }^{1-4}$

Ocorre choque quando o transporte de oxigênio é inadequado para suprir as necessidades metabólicas dos tecidos. Em indivíduos saudáveis, a captação de oxigênio é independente do suprimento de oxigênio. Assim, quando o transporte de oxigênio é agudamente reduzido, a extração de oxigênio aumenta e, consequentemente, o consumo de oxigênio permanece constante. Somente quando o transporte de oxigênio cai abaixo de um ponto crítico, a capacidade de extração de oxigênio é superada e o consumo de oxigênio cai. A partir deste ponto, começa a haver metabolismo anaeróbico e acidose láctica. ${ }^{3,4}$

Em pacientes com choque, frequentemente, há aumento da demanda de oxigênio e a capacidade dos tecidos de extrair oxigênio está diminuída, em decorrência de alterações das células e da microvasculatura. Além disso, o transporte de oxigênio está reduzido em virtude de hipovolemia, disfunção miocárdica e alteração do conteúdo arterial de oxigênio (p.ex., hipóxia, anemia), o que pode resultar em uma dependência patológica do consumo de oxigênio em relação ao transporte, mesmo quando o transporte de oxigênio não está reduzido significativamente. Em modelos animais, a administração de endotoxina resultou em valores críticos de transporte de oxigênio mais elevados, observando-se metabolismo anaeróbico com níveis de transporte considerados adequados para indivíduos saudáveis, o que reforça a necessidade de intervenções te- rapêuticas para aumentar o transporte de oxigênio no choque. ${ }^{3,4}$

Os mecanismos compensatórios para manutenção do débito cardíaco incluem a taquicardia e o aumento da contratilidade cardíaca e do tônus do sistema venoso. Entretanto, em crianças, as respostas cardiovasculares compensatórias diferem daquelas dos adultos. Em crianças pequenas, o débito cardíaco é mais dependente da frequência cardíaca do que do volume de ejeção, em decorrência de menor massa muscular do ventrículo. Assim, a criança compensa a diminuição do débito cardíaco pela taquicardia. Entretanto, como as crianças têm reserva de frequência cardíaca limitada em comparação com os adultos, em virtude da frequência cardíaca basal já elevada, quanto mais jovem a criança, maior a probabilidade de que esta resposta seja inadequada. O aumento do tônus da musculatura das veias resulta em aumento do débito cardíaco pelo aumento da pré-carga, pois mais sangue se move do sistema venoso de alta capacitância para o coração. A falha dos mecanismos compensatórios para a manutenção do débito cardíaco e do transporte de oxigênio resulta em hipóxia tecidual, hipercapnia e acidose. A persistência da acidose e do transporte inadequado de substratos contribui para a diminuição da função miocárdica, que na ausência de intervenções terapêuticas urgentes, pode evoluir para bradicardia e parada cardíaca. ${ }^{1,3}$

A pressão arterial sistêmica é o produto do débito cardíaco pela resistência vascular sistêmica (Equação 4).

Pressão arterial $=$ Débito cardíaco x Resistência vascular sistêmica (Equação 4) bito cardíaco, o aumento da resistência vascular sistêmica mantém a pressão arterial normal. Além disso, a vasoconstrição periférica leva à redistribuição do fluxo sanguíneo dos leitos vasculares não essenciais (pele, musculatura esquelética, rins e esplâncnico) para os órgãos nobres (cérebro, coração, pulmões e adrenais). Esta regulação do tônus vascular pode normalizar a pressão arterial, independentemente do débito cardíaco. É importante salientar que, em crianças, a hipotensão é um sinal tardio e súbito de descompensação cardiovascular. Portanto, a pressão arterial não é um bom indicador da homeostase cardiovascular em pacientes pediátricos. ${ }^{3}$ 


\section{Diagnóstico}

O diagnóstico de choque requer um alto índice de suspeita e o conhecimento das condições predisponentes. A avaliação clínica cuidadosa é essencial ao diagnóstico, devendo-se estar atento às alterações da frequência cardíaca e da pressão arterial, e aos sinais de hipoperfusão tecidual. A Tabela 1 mostra a frequência cardíaca normal nas diversas faixas etárias. Ressalta-se que a taquicardia sinusal é um sinal inespecífico de comprometimento circulatório, pois pode ocorrer em várias situações de estresse (dor, ansiedade e febre). ${ }^{1}$

A hipotensão arterial é definida pelos limites de pressão arterial sistólica de acordo com a idade (Tabela 2). ${ }^{1,2}$

\section{Tabela 1}

Valores de frequência cardíaca normal em batimentos por minuto (bpm) de acordo com a idade (média \pm 2 desvios-padrão)

\begin{tabular}{lc}
\hline Idade & $\begin{array}{c}\text { Frequência cardíaca (bpm) } \\
\text { (média } \pm 2 \text { desvios-padrão) }\end{array}$ \\
\hline Recém-nascido & $140 \pm 50$ \\
$1-6$ meses & $130 \pm 50$ \\
$6-12$ meses & $115 \pm 40$ \\
$1-2$ anos & $110 \pm 40$ \\
$2-6$ anos & $105 \pm 35$ \\
$6-10$ anos & $95 \pm 30$ \\
$>10$ anos & $85 \pm 30$ \\
\hline
\end{tabular}

Fonte: Adaptado de Pediatric Advanced Life Support Provider Manual, 2002.

\section{Tabela 2}

Definição de hipotensão pelos limites de pressão arterial sistólica $(\mathrm{mm} \mathrm{Hg})$ de acordo com a idade

\begin{tabular}{lc}
\hline Idade & Pressão arterial \\
sistólica $(\mathrm{mm} \mathrm{Hg})$
\end{tabular}

Recém-nascidos a termo

(0-28 dias) $\quad<60$

Lactentes (1-12 meses)

$<70$

Crianças 1-10 anos

$<70+(2 \mathrm{x}$ idade em anos $)$

$>10$ anos

$<90$

Fonte: Pediatric Advanced Life Support Provider Manual, 2002.
Ressalta-se que estes limites de pressão arterial se aproximam do percentil 5 da pressão arterial sistólica normal para a idade. Desta forma, estes valores se sobrepõem aos valores normais de 5\% das crianças saudáveis. Além disso, estes limites são valores de referência para crianças normais em repouso. As crianças doentes e sob estresse apresentam frequentemente aumento da pressão arterial. Portanto, a pressão arterial no limite inferior da normalidade pode ser inapropriada para uma criança gravemente doente. ${ }^{1,2,3}$

A palpação dos pulsos dá informações sobre o fluxo sanguíneo e a resistência vascular sistêmica. Os pulsos centrais (femorais, carotídeos e axilares) e periféricos (braquiais, radiais, tibiais posteriores e pediosos) são facilmente palpáveis em crianças saudáveis, com exceção dos pulsos carotídeos em recém-nascidos e lactentes, em decorrência do menor tamanho do pescoço. Os pulsos centrais são, normalmente, mais fortes do que os pulsos perifé ricos, porque possuem maior calibre e estão mais próximos do coração. A vasoconstrição associada ao choque causa aumento da diferença de volume (ou força) entre os pulsos centrais e periféricos. A diminuição da perfusão sistêmica se inicia nas extremidades, com diminuição e desaparecimento dos pulsos periféricos, e progride em direção ao tronco, com enfraquecimento dos pulsos centrais. A pressão de pulso é a diferença entre a pressão arterial sistólica e a diastólica. O aumento da resistência vascular sistêmica em resposta à diminuição do débito cardíaco leva ao estreitamento da pressão de pulso, enquanto que em situações de choque com baixa resistência vascular sistêmica, observa-se alargamento da pressão de pulso. O desaparecimento dos pulsos centrais é um sinal pré-mórbido que indica a necessidade de intervenção muito rápida para evitar parada cardíaca. ${ }^{1-4}$

A diminuição da perfusão cutânea é um sinal precoce de choque. Normalmente, as mãos e os pés encontram-se aquecidos e corados. Quando o débito cardíaco cai, a pele se torna fria, inicialmente nas extremidades e, subsequentemente, no tronco. Lividez reticular, palidez, cianose de extremidades e prolongamento do tempo de enchimento capilar ( $>2$ segundos) também indicam perfusão cutânea diminuída. Em recém-nascidos, a vasoconstrição intensa produz coloração acinzentada na pele, enquanto que em crianças mais velhas, observa-se palidez acentuada. Os sinais de hipoperfusão cerebral incluem alteração do nível de consciência (irritabilidade, agitação, letargia, 
coma), hipotonia, crises convulsivas e alterações pupilares. Para avaliação rápida das funções corticais cerebrais recomenda-se o uso da Escala de Resposta Pediátrica "AVDN": $\mathrm{A}=$ alerta; $\mathrm{V}=$ responsivo à voz; $\mathrm{D}=$ responsivo à dor; $\mathrm{N}=$ não responsivo. Outros sinais de má perfusão orgânica incluem a diminuição do débito urinário $(<1 \mathrm{~mL} / \mathrm{kg} / \mathrm{h}$, em recém-nascidos e lactentes ou $<12 \mathrm{~mL} / \mathrm{m}^{2} / \mathrm{h}$, em crianças maiores e adolescentes) e acidose láctica. ${ }^{1-4}$

\section{Classificação}

\section{Segundo o estado fisiológico}

O choque é classificado como compensado ou descompensado, de acordo com seu efeito na pressão arterial. O choque é definido como compensado quando os mecanismos compensatórios são capazes de manter a pressão arterial normal, ou seja, o paciente apresenta sinais e sintomas de perfusão tecidual inadequada (acidose láctica, oligúria, alteração do nível de consciência), mas a pressão arterial sistólica é normal. O choque é classificado como descompensado quando os sinais de choque se associam com hipotensão sistólica. ${ }^{1}$

O choque é classificado segundo o débito cardíaco em hipodinâmico ou frio e hiperdinâmico ou quente. $\mathrm{O}$ choque hipodinâmico ou frio se associa a baixo débito cardíaco e ocorre em crianças com choque hipovolêmico, séptico e cardiogênico. Os mecanismos compensatórios causam aumento da resistência vascular sistêmica, observando-se pele fria e marmórea, pulsos finos e perfusão periférica diminuída (tempo de enchimento capilar $>2$ segundos). $\mathrm{O}$ choque hiperdinâmico ou quente se associa a alto débito cardíaco e baixa resistência vascular sistêmica e ocorre no choque anafilático e em algumas crianças com choque séptico. Caracteriza-se por extremidades quentes, avermelhadas, com alargamento da pressão de pulso e perfusão periférica rápida. Nestas situações, ocorre choque a despeito do débito cardíaco elevado, porque o fluxo sanguíneo é distribuído inadequadamente. Alguns tecidos recebem fluxo sanguíneo insuficiente (p.ex., a circulação esplâncnica), enquanto outros (p.ex., pele e músculos) recebem fluxo sanguíneo excessivo em relação às suas demandas metabólicas. ${ }^{1,2}$

\section{Segundo a etiologia}

O choque é classificado segundo a etiologia em hipovolêmico, cardiogênico, distributivo, obstrutivo e séptico. Entretanto, esta classificação representa uma simplificação, porque as etiologias frequentemente se sobrepõem. ${ }^{1-4}$

\section{Choque hipovolêmico}

Caracteriza-se por volume intravascular inadequado relativo ao espaço vascular. A hipovolemia é a principal causa de choque em crianças, resultante de desidratação, hemorragia e perdas para o terceiro espaço, decorrentes do aumento da permeabilidade capilar (p.ex., sepse, queimaduras). A hipovolemia relativa ocorre em situações de vasodilatação sistêmica com aumento da capacidade vascular, como sepse e anafilaxia.

A diminuição do volume intravascular leva à diminuição do retorno venoso e da pré-carga, e, consequentemente, do volume sistólico e do débito cardíaco. A ativação dos barorreceptores periféricos e centrais produz a liberação de catecolaminas, resultando em aumento da frequência cardíaca e da resistência vascular sistêmica, que constituem os mecanismos compensatórios para a manutenção da pressão arterial. A hipotensão é um achado tardio. Em crianças com hemorragia, a hipotensão geralmente ocorre com perda aguda de mais de $25 \%$ a $30 \%$ do volume sanguíneo circulante (Figura 1). ${ }^{1}$

Os sinais clínicos do choque hipovolêmico são: Taquicardia, pressão arterial normal (choque compensado) ou diminuída (choque descompensado), diminui-

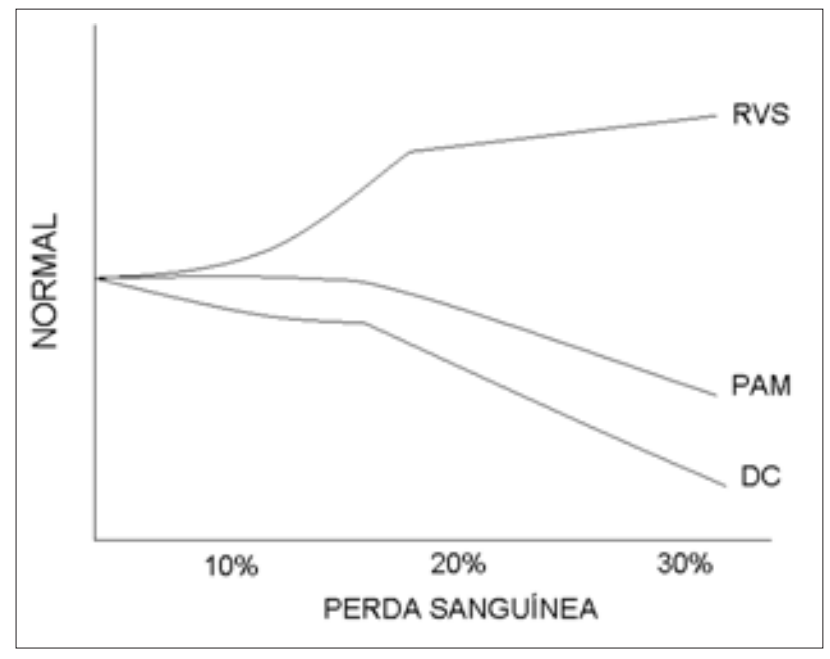

Figura 1: Evolução da resistência vascular sistêmica (RVS), da pressão arterial média (PAM) e do débito cardíaco (DC) de acordo com a porcentagem de volume sanguíneo circulante perdido. Fonte: Adaptado de Pediatric Advanced Life Support - Provider Manual, 2002. 
ção da pressão de pulso, pulsos finos, tempo de enchimento capilar prolongado ( $>2$ segundos), pele fria, pálida ou marmórea, diaforese, alteração do estado mental e oligúria.

\section{Choque cardiogênico}

Resulta de disfunção miocárdica. As causas incluem as cardiomiopatias, os distúrbios do ritmo, as cardiopatias congênitas e as lesões traumáticas do coração (Tabela 3$) \cdot \cdot^{1-4}$

O choque cardiogênico se caracteriza por baixo débito cardíaco e alta resistência vascular sistêmica. A diminuição do débito cardíaco leva à liberação de mediadores neurohumorais, que resulta em aumento da resistência vascular sistêmica e da pós-carga do ventrículo esquerdo. Desta forma, os mecanismos

\section{Tabela 3}

Causas de choque cardiogênico em crianças

\section{Cardiomiopatias}

- Eventos hipóxico-isquêmicos

- Parada cardiocirculatória, pós-circulação extracorpórea, anomalia de artérias coronárias

- Infecciosas

- Miocardite viral, sepse

- Metabólicas

- Acidose, hipocalcemia, doenças de depósito

- Doenças do tecido conjuntivo

- Lúpus eritematoso sistêmico, doença de Kawasaki, febre reumática

\section{Distúrbios do ritmo}

- Taquicardia supraventricular

- Taquicardia ventricular

- Bloqueio atrioventricular

\section{Cardiopatias congênitas}

- Obstruções da via de saída do ventrículo esquerdo

- Interrupção do arco aórtico, coarctação da aorta, estenose aórtica crítica, síndrome do coração esquerdo hipoplásico

\section{Trauma}

- Contusão miocárdica

- Aneurismas

- Ruptura valvar compensatórios do choque cardiogênico podem ter efeitos deletérios, porque o aumento da pós-carga do ventrículo esquerdo pode deteriorar ainda mais a função miocárdica. ${ }^{1-4}$

O reconhecimento do choque cardiogênico se inicia pela história cuidadosa. Relatos típicos incluem aumento do esforço respiratório, dificuldades de alimentação, dispneia às mamadas, sudorese excessiva, baixo ganho pôndero-estatural e infecções respiratórias frequentes (em crianças com cardiopatias congênitas com hiperfluxo pulmonar). Ao exame físico, podese observar taquicardia, ritmo de galope, taquipneia, extremidades frias, pulsos finos, cianose, diaforese, estertores crepitantes, sibilos (pelo edema pulmonar "asma cardíaca"), hepatomegalia, estase jugular (em crianças maiores) e edema periférico (manifestação tardia de insuficiência cardíaca em crianças). Em recém-nascidos, as obstruções congênitas da via de saída do ventrículo esquerdo se manifestam por choque cardiogênico nas duas primeiras semanas de vida, por ocasião do fechamento do canal arterial. Em crianças com coarctação de aorta grave ou interrupção do arco aórtico, além dos sinais de choque, observa-se diferencial importante de pressão arterial e de intensidade dos pulsos entre os membros superiores e os inferiores (pressão arterial mais baixa nos membros inferiores que nos membros superiores e diminuição da amplitude ou ausência dos pulsos femorais). Como os mecanismos compensatórios do choque cardiogênico podem deteriorar ainda mais a função miocárdica, a fase compensada do choque cardiogênico pode não ser observada; geralmente, o paciente apresenta-se hipotenso. ${ }^{1,2}$

O diagnóstico clínico deve ser suplementado pela radiografia de tórax, cujos achados típicos são cardiomegalia e congestão vascular pulmonar, além do eletrocardiograma e da ecocardiografia, que dão o diagnóstico da causa do choque. Recentemente, marcadores bioquímicos de lesão celular e disfunção miocárdica têm sido utilizados, como a troponina I cardíaca e o peptídeo natriurético tipo B (BNP), para o diagnóstico e a monitoração de pacientes com choque cardiogênico. ${ }^{5}$

\section{Choque distributivo}

Caracteriza-se pela distribuição inadequada de sangue aos tecidos que resulta em má perfusão tecidual, geralmente secundária a alterações do tônus vasomotor. As causas de choque distributivo incluem anafilaxia, anestesia espinhal ou epidural, secção de 
medula, disfunção grave do cérebro e do tronco cerebral e uso inapropriado de vasodilatador. ${ }^{1,2}$

No choque anafilático, há vasodilatação sistêmica, aumento da permeabilidade capilar com hipovolemia relativa e vasoconstricção pulmonar. Os sinais e sintomas incluem agitação, náuseas e vômitos, urticária, angioedema, desconforto respiratório com estridor ou sibilos, hipotensão e taquicardia. ${ }^{1,2}$

No choque neurogênico, a perda da inervação simpática da musculatura lisa da parede vascular resulta em vasodilatação. O paciente apresenta hipotensão com alargamento da pressão de pulso, sem taquicardia compensatória, porque a inervação simpática do coração também está comprometida. ${ }^{1,2,3}$

\section{Choque obstrutivo}

Caracteriza-se por débito cardíaco adequado na vigência de volume intravascular e função miocárdica normais em decorrência de obstrução mecânica à entrada e/ou saída de sangue do coração. As causas de choque obstrutivo são pneumotórax hipertensivo, tamponamento cardíaco e embolia pulmonar maciça. ${ }^{1,2}$

No tamponamento cardíaco, a compressão do coração secundária ao aumento da pressão intrapericárdica impede o retorno venoso sistêmico e pulmonar e reduz o enchimento ventricular. Consequentemente, o débito cardíaco cai. Em crianças, geralmente, ocorre tamponamento cardíaco após trauma penetrante ou cirurgia cardíaca. As manifestações clínicas são abafamento das bulhas cardíacas, pulso paradoxal (diminuição da pressão sistólica mais de $10 \mathrm{~mm}$ Hg durante a inspiração) e distensão das veias do pescoço. O eletrocardiograma mostra complexos QRS de baixa amplitude e o diagnóstico definitivo é feito pelo ecocardiograma. $\mathrm{Na}$ ausência de tratamento, o tamponamento cardíaco resulta em parada cardíaca caracterizada por atividade elétrica sem pulso. ${ }^{1}$

No pneumotórax hipertensivo, a compressão do pulmão causa falência respiratória, e a compressão das estruturas mediastinais (coração e grandes vasos) leva à diminuição do retorno venoso e do débito cardíaco. Deve-se suspeitar de pneumotórax hipertensivo em vítimas de trauma torácico ou em pacientes intubados que deterioram subitamente durante a ventilação com pressão positiva (bolsa-valva-máscara ou ventilação mecânica). Os sinais clínicos são hiperressonância à percussão com diminuição do murmúrio vesicular no lado afetado, distensão das veias do pescoço, desvio da traqueia para o lado contralateral, deterioração rápida da perfusão e taquicardia, com evolução rápida para bradicardia e parada cardíaca (atividade elétrica sem pulso). ${ }^{1,2}$

A embolia pulmonar é relativamente rara em crianças, sendo mais frequentemente observada em adolescentes e adultos. Resulta em desequilíbrio ventilação-perfusão, hipóxia e aumento da resistência vascular pulmonar que leva à insuficiência cardíaca direita e diminuição do débito cardíaco. ${ }^{1}$

\section{Síndrome da resposta inflamatória sistêmica(SRIS), sepse e choque séptico}

A SRIS caracteriza a resposta inflamatória independente da causa. É definida pela presença de duas ou mais das seguintes condições, uma das quais deve ser alteração da temperatura ou da contagem de leucócitos:

- Febre ou hipotermia

- Taquicardia

- Taquipneia

- Hemograma com leucocitose, leucopenia ou desvio à esquerda.

Sepse é a SRIS na presença de infecção (suspeita ou confirmada). Choque séptico é definido pela sepse associada a alterações da perfusão sistêmica: Alteração do nível de consciência (irritabilidade, sonolência), oligúria (diurese $<1 \mathrm{~mL} / \mathrm{kg} / \mathrm{h}$ ou $<12 \mathrm{~mL} /$ $\mathrm{m}^{2} / \mathrm{h}$ ) e acidose láctica. $\mathrm{O}$ choque séptico pode ser hipodinâmico ou frio (com baixo débito cardíaco), caracterizado por extremidades frias, tempo de enchimento capilar $>2$ segundos e diminuição da pressão de pulso, ou hiperdinâmico ou quente (com alto débito cardíaco e baixa resistência vascular sistêmica), caracterizado por extremidades quentes, avermelhadas e alargamento da pressão de pulso. A hipotensão não é necessária para o diagnóstico clínico de choque séptico em crianças; entretanto, em crianças com suspeita clínica de infecção sua presença é confirmatória (choque séptico descompensado). ${ }^{1-4,6}$

O choque séptico pode ser considerado uma combinação de vários tipos de choque, incluindo o hipovolêmico, o cardiogênico e o distributivo. A hipovolemia é resultante de lesão endotelial e do aumento da permeabilidade capilar causados pela resposta inflamatória sistêmica. Além disso, muitas crianças com choque séptico têm história de diminuição da ingestão e aumento das perdas (p.ex., diarreia, vômitos), e podem também apresentar hipovolemia relativa causada por vasodilatação. A disfunção miocárdica é comum em crianças com choque séptico, em decorrência da lesão inflamatória ou tóxica causada por me- 
diadores inflamatórios, como o fator de necrose tumoral-alfa (TNF- $\alpha$ ) a presença de fatores depressores do miocárdio circulantes (TNF- $\alpha$, óxido nítrico e interleucina-1) e down-regulação dos receptores $\beta$ adrenérgicos. A alteração do tônus vascular causada pelos mediadores inflamatórios envolve dilatação e constrição da microvasculatura, que resultam em desrregulação do fluxo sanguíneo vascular, hipóxia e falência orgânica. ${ }^{1-4,6}$ Ao contrário do que ocorre na maioria dos adultos, que apresenta choque hiperdinâmico (com alto débito cardíaco e baixa resistência vascular sistêmica), aproximadamente $80 \%$ das crianças com choque séptico têm baixo débito cardíaco. ${ }^{7}$

\section{Tratamento do choque}

$\mathrm{O}$ reconhecimento precoce e o tratamento agressivo dos vários tipos de choque podem melhorar o desfecho. Portanto, a velocidade da intervenção é crucial. Faz toda a diferença ter o conhecimento e as habilidades necessárias para atender a criança gravemente doente no início do quadro, pois quanto maior o intervalo de tempo entre o evento precipitante e o início da ressuscitação, pior o desfecho. ${ }^{1}$

O objetivo do tratamento do choque é o restabelecimento eficaz da perfusão e da oxigenação tecidual, evidenciado por:

- Tempo de enchimento capilar menor ou igual a 2 segundos

- Pulsos normais

- Extremidades aquecidas

- Diurese $>1 \mathrm{~mL} / \mathrm{kg} / \mathrm{h}$ ou $>12 \mathrm{~mL} / \mathrm{m}^{2} / \mathrm{h}$

- Nível de consciência normal

- Pressão arterial normal para a idade

- Saturação venosa central de oxigênio maior ou igual a $70 \%$

O manejo inicial do choque consiste nos princípios básicos de ressuscitação: manutenção das vias aéreas, ventilação e oxigenação. Após o posicionamento adequado da cabeça e a aspiração das vias aéreas, deve-se administrar oxigênio por meio de dispositivos de alto fluxo (máscara não-reinalante) a todas as crianças com choque. A intubação traqueal deve ser precoce, com o objetivo de diminuir o consumo de oxigênio pelos músculos respiratórios, sendo indicada nas seguintes situações: aumento do trabalho respiratório, hipoventilação, diminuição do nível de consciência e instabilidade hemodinâmica grave. . $^{1-4,6}$

$\mathrm{O}$ acesso vascular deve ser estabelecido rapidamente. A primeira escolha é a punção de veia peri- férica - de preferência, duas veias calibrosas, utilizando cateteres curtos e grossos (tipo cateter sobre agulha). Caso o acesso venoso periférico não seja obtido prontamente em poucos minutos, a via intraóssea deve ser estabelecida, lembrando que ela pode ser obtida rapidamente em pacientes de todas as idades (inclusive em adultos). ${ }^{1,2}$

Imediatamente após a obtenção do acesso vascular, inicia-se a ressuscitação hídrica, cujo objetivo é adequar a volemia e restaurar a perfusão tecidual. Em pacientes com choque hipovolêmico, distributivo ou séptico, deve-se, inicialmente, administrar bolus de cristaloide (soro fisiológico a $0,9 \%$ ), $20 \mathrm{~mL} / \mathrm{kg}$ em 5 a 20 minutos. Durante a ressuscitação hídrica, a criança deve ser reavaliada continuamente, observando-se a frequência cardíaca, a pressão arterial, os pulsos, o tempo de enchimento capilar, o estado mental, o débito urinário e a presença de sinais de sobrecarga de volume (estertores à ausculta pulmonar, ritmo de galope e hepatomegalia). Enquanto se procede à ressuscitação hídrica, deve-se puncionar um acesso venoso central, para monitorização da pressão venosa central e posterior infusão de drogas vasoativas, se necessário. Na ausência de sinais de sobrecarga hídrica, deve-se prosseguir com expansões de volume, até a adequação da volemia (pressão venosa central maior ou igual a 8 a $12 \mathrm{~mm} \mathrm{Hg}$ ). ${ }^{6,8}$

As crianças com choque séptico usualmente necessitam de 40 a $60 \mathrm{~mL} / \mathrm{kg}$ de ressuscitação hídrica na primeira hora. ${ }^{9}$ Em pacientes com cetoacidose diabética que se apresentam com choque hipovolêmico, a ressuscitação hídrica deve ser realizada mais lentamente (10 a $20 \mathrm{~mL} / \mathrm{kg}$ de soro fisiológico a $0,9 \%$ em 1 hora), pelo risco de edema cerebral associado à administração rápida de fluido. Em pacientes com choque cardiogênico, deve-se realizar a ressuscitação hídrica cuidadosamente, com soro fisiológico a $0,9 \% 5$ a 10 $\mathrm{mL} / \mathrm{kg}$ em 15 a 20 minutos, com o objetivo de melhorar o débito cardíaco pela otimização da pré-carga. ${ }^{1,2,3}$

Com relação ao tipo de fluido a ser administrado durante a ressuscitação hídrica, se cristaloide ou coloide, algumas considerações devem ser feitas. As vantagens das soluções cristaloides são o baixo custo, a ausência de exposição a produtos do sangue e a ampla disponibilidade; entretanto, como apenas $25 \%$ do volume administrado permanecem no intravascular, podem ser necessários grandes volumes para ressuscitação, o que aumenta o risco de edema. Por outro lado, as soluções coloides permitem melhor expansão com menores volumes, pois suas moléculas 
são relativamente impermeáveis à membrana capilar, e, consequentemente, há menor extravasamento. Entretanto, em situações com lesão endotelial, como na sepse, pode haver extravasamento de $15 \%$ a $20 \%$ do volume administrado, resultando em aumento da pressão oncótica no espaço intersticial e piora do edema tissular. Além disso, as soluções coloides são de alto custo. ${ }^{1,2,3}$ Uma meta-análise recente concluiu que, atualmente, não há evidências de que a ressuscitação hídrica com coloides reduza o risco de morte quando comparados com cristaloides. ${ }^{10} \mathrm{Na}$ prática, no Hospital das Clínicas da Faculdade de Medicina de Ribeirão Preto da Universidade de São Paulo, utiliza-se solução de albumina $5 \% 10$ a $20 \mathrm{~mL} / \mathrm{kg}$, caso a criança persista com sinais de choque após ter recebido 60 $\mathrm{mL} / \mathrm{kg}$ de cristaloide e ainda necessite de fluido adicional para a adequação da volemia. ${ }^{8}$

Recomenda-se transfusão de concentrado de hemácias $(10-15 \mathrm{~mL} / \mathrm{kg})$ para pacientes pediátricos vítimas de trauma com choque hemorrágico, quando houver persistência dos sinais de choque ou instabilidade hemodinâmica após a administração de 40 a 60 $\mathrm{mL} / \mathrm{kg}$ de cristaloide. Além disso, com o objetivo de otimizar o transporte de oxigênio, deve-se administrar concentrado de hemácias se a concentração de hemoglobina estiver abaixo de $10 \mathrm{~g} / \mathrm{dL}$ em crianças com qualquer tipo de choque. ${ }^{1,2}$

$\mathrm{O}$ tratamento com drogas vasoativas deve ser iniciado se o paciente ainda tiver sinais de choque, mesmo após a adequação da volemia, (Tabela 4). ${ }^{1,8}$

Em crianças com choque séptico, a droga de escolha é a dopamina. Se não houver melhora com dopamina, deve-se iniciar epinefrina em crianças com choque frio ou norepinefrina em crianças com choque quente. ${ }^{6,8}$ Ressalta-se a importância da antibioticote- rapia específica, que deve ser iniciada na primeira hora de tratamento, ${ }^{11}$ além da correção dos distúrbios metabólicos, especialmente a acidose, a hipoglicemia e a hipocalcemia, que contribuem para a disfunção miocárdica. ${ }^{6} \mathrm{~A}$ administração de corticosteroides está indicada em crianças com risco de insuficiência adrenal (púrpura fulminante, uso crônico de corticosteroides, doença do sistema nervoso central) com choque refratário às catecolaminas. ${ }^{6,8}$ Utiliza-se a hidrocortisona em dose de estresse (ataque $50 \mathrm{mg}$ em lactentes, 100 a $150 \mathrm{mg}$ em crianças maiores e adolescentes; manutenção $100 \mathrm{mg} / \mathrm{m}^{2} /$ dia, $6 / 6$ horas). ${ }^{8}$

Em crianças com choque cardiogênico, o tratamento farmacológico consiste no uso de inotrópicos e vasodilatadores. A milrinona ou a dobutamina são as drogas de escolha, pois ambas têm propriedades inotrópicas e vasodilatadoras. O uso de diurético (furosemida) está indicado em pacientes com edema pulmonar ou congestão venosa sistêmica; porém, o diurético deve ser administrado apenas após a restauração da perfusão sistêmica e a normalização da pressão arterial. No manejo do choque cardiogênico, as medidas que visam minimizar as demandas de oxigênio são fundamentais, incluindo o suporte ventilatório precoce, o uso de sedativos e analgésicos e a manutenção da temperatura corpórea normal. Salienta-se também a importância de manter a homeostase metabólica ( $\mathrm{pH}$, glicose, cálcio e magnésio), corrigir a anemia e tratar as arritmias. ${ }^{1-4}$

O suporte farmacológico do choque anafilático inclui o uso de epinefrina, anti-histamínicos (bloqueador $\mathrm{H}_{1}$ e $\mathrm{H}_{2}$ ) e corticosteroides. O tratamento do choque obstrutivo consiste no manejo da causa específica (p.ex., drenagem pericárdica em crianças com tamponamento cardíaco, descompressão do pneumotórax

\section{Tabela 4}

Drogas vasoativas

\begin{tabular}{|c|c|c|}
\hline Droga & Dose & Efeitos \\
\hline Dopamina & $5-15 \mu \mathrm{g} / \mathrm{kg} / \mathrm{min}$ & $\begin{array}{l}\text { Inotrópico }(5-10 \mu \mathrm{g} / \mathrm{kg} / \mathrm{min}) \\
\text { Vasoconstrictor }(>10 \mu \mathrm{g} / \mathrm{kg} / \mathrm{min})\end{array}$ \\
\hline Epinefrina & $0,01-1 \mu \mathrm{g} / \mathrm{kg} / \mathrm{min}$ & $\begin{array}{l}\text { Inotrópico }(0,01-0,3 \mu \mathrm{g} / \mathrm{kg} / \mathrm{min}) \\
\text { Vasoconstritor }(>0,3 \mu \mathrm{g} / \mathrm{kg} / \mathrm{min})\end{array}$ \\
\hline Norepinefrina & $0,01-1 \mu \mathrm{g} / \mathrm{kg} / \mathrm{min}$ & $\begin{array}{l}\text { Inotrópico }(0,01-0,2 \mu \mathrm{g} / \mathrm{kg} / \mathrm{min}) \\
\text { Vasoconstrictor }(>0,2 \mu \mathrm{g} / \mathrm{kg} / \mathrm{min})\end{array}$ \\
\hline Dobutamina & $5-20 \mu \mathrm{g} / \mathrm{kg} / \mathrm{min}$ & Inotrópico e vasodilatador sistêmico e pulmonar \\
\hline Milrinona & $0,2-1 \mu \mathrm{g} / \mathrm{kg} / \mathrm{min}$ & Inotrópico e vasodilatador sistêmico e pulmonar \\
\hline
\end{tabular}


com agulha seguida de colocação de dreno pleural em pacientes com pneumotórax hipertensivo e uso de trombolíticos em crianças com embolia pulmonar maciça). ${ }^{1,2}$

\section{Casos clínicos}

\section{Caso 1}

Pré-escolar de 2 anos, $12 \mathrm{~kg}$, tem história de febre alta $\left(40^{\circ} \mathrm{C}\right)$ há 12 horas e apatia; há 2 horas, a criança ficou mais sonolenta e a mãe notou surgimento de manchas avermelhadas pelo corpo. Ao exame físico, a criança encontra-se em mau estado geral, agitada e confusa. Pele com sufusões hemorrágicas em tronco e membros. Frequência respiratória 42 ipm, frequência cardíaca 150 bpm, pressão arterial 60/ 30 $\mathrm{mm} \mathrm{Hg}$, pulsos centrais e periféricos finos, extremidades frias, tempo de enchimento capilar 4 segundos.

\section{Pergunta-se:}

\section{Quais são os diagnósticos?}

Resposta: Choque séptico frio descompensado e meningococcemia.

Comentário: A criança apresenta sepse (síndrome da resposta inflamatória sistêmica caracterizada por febre, taquicardia e taquipneia, e suspeita de infecção pela presença de febre com deterioração rápida e aparecimento de sufusões hemorrágicas pelo corpo, sugestivos de meningococcemia). Há sinais de comprometimento da perfusão sistêmica: alteração do nível de consciência (agitada, confusa), pulsos finos, extremidades frias, tempo de enchimento capilar maior que 2 segundos. Portanto, o quadro é compatível com choque séptico hipodinâmico ou frio. A pressão arterial sistólica mínima para a idade é de $70+(2$ x idade em anos $)=74 \mathrm{~mm} \mathrm{Hg}$; desta forma, como a criança encontra-se hipotensa, o choque séptico é classificado como descompensado.

\section{Qual é o tratamento inicial?}

Resposta: Abertura das vias aéreas, oxigênio por máscara não-reinalante, acesso vascular periférico ou intraósseo, expansão com soro fisiológico $0,9 \%$ $240 \mathrm{~mL}(20 \mathrm{~mL} / \mathrm{kg})$ em 5-20 minutos.

3. Após ter realizado 3 expansões com soro fisiológico $0,9 \%$, total $720 \mathrm{~mL}(60 \mathrm{~mL} / \mathrm{kg})$ em 1 hora, a criança persiste com sinais de choque. Foi puncionada veia central e a pressão venosa central é de $12 \mathrm{~mm} \mathrm{Hg}$. O que deve ser feito agora?

Resposta: Iniciar dopamina 5 microgramas/kg/min.

\section{Caso 2}

Lactente de 1 ano, $10 \mathrm{~kg}$, tem história de febre alta $\left(39^{\circ} \mathrm{C}\right)$ há 3 dias e desconforto respiratório, com piora progressiva. Mãe relata diminuição da diurese há 1 dia e dificuldade de alimentação, com piora da dispneia às mamadas e vômitos ocasionais. Nega diarreia. Ao exame físico, a criança encontra-se em mau estado geral, pálida, sonolenta, com saliva escassa. Frequência respiratória $80 \mathrm{ipm}$. Saturação de $\mathrm{O}_{2}$ $89 \%$ em ar ambiente. Murmúrio vesicular diminuído globalmente, com sibilos expiratórios disseminados e estertores bolhosos ocasionais, bilateralmente. Tiragem intercostal e subcostal. Batimento de asas do nariz. Respira com a boca aberta. Ritmo cardíaco regular em 2 tempos, bulhas hipofonéticas, sem sopros. Frequência cardíaca 180 bpm. Pressão arterial 80/50 mm $\mathrm{Hg}$. Pulsos centrais e periféricos finos. Pele fria e rendilhada. Tempo de enchimento capilar 5 segundos. Abdome: Fígado a $5 \mathrm{~cm}$ do RCD, endurecido. A radiografia simples de tórax evidencia aumento da área cardíaca e velamento pulmonar difuso. A concentração plasmática da fração amino terminal do peptídeo natriurético do tipo B (NT-pró-BNP) é de 5000 pg/ $\mathrm{mL}$ (normal até $125 \mathrm{pg} / \mathrm{mL}$ ).

\section{Pergunta-se:}

1. Quais são os diagnósticos?

Resposta: Choque cardiogênico compensado secundário à provável miocardite viral.

Comentário: A história de desconforto respiratório, dificuldade de alimentação e piora da dispneia às mamadas e o exame físico que mostra sinais de congestão venosa pulmonar (taquipneia, sibilos e estertores) e sistêmica (hepatomegalia), associados aos sinais de má perfusão tecidual (sonolência, diminuição da diurese, pulsos finos, tempo de enchimento capilar $>2$ segundos) são compatíveis com o diagnóstico de choque cardiogênico. Como a pressão arterial está normal, o choque é compensado. $\mathrm{O}$ aumento da área cardíaca, os sinais de edema pulmonar na radiografia de tórax e a concentração plasmática aumentada do NT-pró-BNP confirmam a suspeita clínica. O diagnóstico etiológico deve ser 
confirmado pelo eletrocardiograma e pela ecocardiografia.

2. Qual é o tratamento inicial?

Resposta: Abertura das vias aéreas, oxigênio por máscara não-reinalante, acesso vascular periférico ou intraósseo, expansão com soro fisiológico $0,9 \%$ $50 \mathrm{~mL}(5 \mathrm{~mL} / \mathrm{kg})$ em 15-20 minutos.

3. Após a expansão, a criança persiste com sinais de choque e há piora do desconforto respiratório, com sibilos e estertoração pulmonar difusa e aumento do fígado (palpável a $7 \mathrm{~cm}$ do RCD). O que deve ser feito agora?

Resposta: Iniciar dobutamina 5 microgramas $/ \mathrm{kg} / \mathrm{min}$ ou milrinona 0,375 microgramas $/ \mathrm{kg} / \mathrm{min}$ e, após a normalização da perfusão tecidual (pulsos centrais e periféricos amplos, tempo de enchimento capilar menor ou igual a 2 segundos, extremidades aquecidas), administrar diurético (furosemida $1 \mathrm{mg} / \mathrm{kg}$ ).

\section{Caso 3}

Pré-escolar de 3 anos, 14 kg, vítima de atropelamento por automóvel há 1 hora, chegou à unidade básica de saúde 15 minutos após o acidente, consciente (Escala de Coma de Glasgow 15), com ferimento extenso em couro cabeludo e exposição da calota craniana. Realizado curativo compressivo e a criança foi encaminhada à sala de trauma de hospital de referência após 30 minutos. Ao exame físico de entrada na sala de trauma, a criança encontra-se em mau estado geral, descorada $++/ 4+$, com frequência respiratória de $60 \mathrm{ipm}$, frequência cardíaca $180 \mathrm{bpm}$, pressão arterial 60/30 mm Hg, Escala de Coma de Glasgow 10. Pulsos centrais e periféricos finos, tempo de enchimento capilar 6 segundos. O abdome apresenta tensão aumentada, é doloroso à palpação, e os ruídos hidroaéreos estão diminuídos. O curativo no segmento cefálico está bastante molhado com sangue.

\section{Pergunta-se:}

1. Quais são os diagnósticos?

Resposta: Choque hipovolêmico (hemorrágico) descompensado secundário a lesão do couro cabeludo e trauma abdominal fechado.

Comentário: A criança politraumatizada apresenta-se descorada, com hemorragia visível (ferimento em couro cabeludo) e há sinais de má perfusão tecidual (pulsos finos, tempo de enchimento capilar $>2$ segundos, diminuição do nível de consciência - Escala de Coma de Glasgow 10); portanto, o quadro é compatível com choque hipovolêmico (hemorrágico). A pressão sistólica mínima para a idade é de $70+$ $(2 \mathrm{x}$ idade em anos $)=76 \mathrm{~mm} \mathrm{Hg}$; deste modo, o choque é classificado como descompensado. Além da hemorragia em couro cabeludo, há provável sangramento intra-abdominal, pois o abdome está tenso, doloroso e os ruídos hidroaéreos estão diminuídos.

2. Qual é o tratamento inicial?

Resposta: Abertura das vias aéreas, oxigênio por máscara não-reinalante, acesso vascular periférico ou intraósseo, expansão com soro fisiológico $0,9 \%$ $280 \mathrm{~mL}(20 \mathrm{~mL} / \mathrm{kg})$ em 5-10 minutos.

3. Após duas expansões (total $560 \mathrm{~mL}$ de soro fisiológico $0,9 \%$ (40 mL/kg) em 20 minutos), a criança persiste com sinais de choque. $\mathrm{O}$ que deve ser feito agora?

Resposta: Transfusão de concentrado de hemácias $10-15 \mathrm{~mL} / \mathrm{kg}$ e controle do sangramento (sutura do ferimento em couro cabeludo e laparotomia exploradora).

\section{ABSTRACT}

This paper presents a review of the definition, classification, pathophysiology and initial management of the different types of shock in children. Early recognition and aggressive treatment of shock in a timely manner are essential to the prevention of cardiopulmonary arrest and outcome improvement.

Keywords: Shock. Child. Diagnosis. Fluid Resuscitation. Hemodynamic Support. 


\section{Referências Bibliográficas}

1. American Heart Association. PALS - Pediatric Advanced Life Support - Provider Manual; 2002.

2. American Heart Association. PALS - Pediatric Advanced Life Support - Provider Manual; 2006.

3. McKiernan CA, Lieberman SA. Circulatory shock in children An overview. Pediatrics in Review 2005; 26:445-53.

4. Nadel S, Kissoon NT, Ranjit S. Recognition and initial management of shock. In: Nichols DG, editor. Rogers' textbook of pediatric intensive care. 4th ed. Philadelphia: Lippincott Williams \& Wilkins; 2008. p. 372-83.

5. Domico M, Checchia PA. Biomonitors of cardiac injury and performance: B-type natriuretic peptide and troponin as monitors of hemodynamics and oxygen transport balance. Pediatr Crit Care Med 2011; 12:S33-S42.

6. Brierley J, Carcillo JA, Choong K, Cornell T, Decaen A, Deymann A, et al. 2007 American College of Critical Care Medicine clinical practice parameters for hemodynamic support of pediatric and neonatal septic shock. Crit Care Med 2009; 37: 666-88.
7. Ceneviva G, Paschall JA, Maffei F, Carcillo JA. Hemodynamic support in fluid-refractory pediatric septic shock. Pediatrics 1998, 102:e19.

8. Nóbrega RF. Sepse e choque séptico. In: Carlotti APCP, editora. Manual de Rotinas de Terapia Intensiva Pediátrica. $2^{\text {a }}$ ed. São Paulo: Tecmedd; 2007. p. 159-65.

9. Carcillo JA, Davis AL, Zaritsky A. Role of early fluid resuscitation in pediatric septic shock. JAMA 1991; 266:1242-5.

10. Perel P, Roberts I. Colloids versus crystalloids for fluid resuscitation in critically ill patients. Cochrane Database of Systematic Reviews 2011, Issue 3. Art.No.: CD000567. DOI: 10.1002/ 14651858. CD000567. pub4.

11. Kumar A, Roberts D, Wood KE, Light B, Parrillo JE, Sharma S, et al. Duration of hypotension before initiation of effective antimicrobial therapy is the critical determinant of survival in human septic shock. Crit Care Med 2006; 34: 1589-96. 